\title{
Changes in Simple Spike Activity of Some Purkinje Cells in the Oculomotor Vermis during Saccade Adaptation Are Appropriate to Participate in Motor Learning
}

\author{
Yoshiko Kojima, ${ }^{1,2}$ Robijanto Soetedjo, ${ }^{1,2}$ and Albert F. Fuchs ${ }^{1,2}$ \\ ${ }^{1}$ Department of Physiology and Biophysics and ${ }^{2}$ Washington National Primate Research Center, University of Washington, Seattle, Washington 98195-7330
}

\begin{abstract}
Adaptation of saccadic eye movements provides an excellent motor learning model to study theories of neuronal plasticity. When primates make saccades to a jumping target, a backward step of the target during the saccade can make it appear to overshoot. If this deception continues for many trials, saccades gradually decrease in amplitude to go directly to the back-stepped target location. We used this adaptation paradigm to evaluate the Marr-Albus hypothesis that such motor learning occurs at the Purkinje (P)-cell of the cerebellum. We recorded the activity of identified P-cells in the oculomotor vermis, lobules VIc and VII. After documenting the on and off error directions of the complex spike activity of a P-cell, we determined whether its saccade-related simple spike (SS) activity changed during saccade adaptation in those two directions. Before adaptation, 57 of 61 P-cells exhibited a clear burst, pause, or a combination of both for saccades in one or both directions. Sixty-two percent of all cells, including two of the four initially unresponsive ones, behaved differently for saccades whose size changed because of adaptation than for saccades of similar sizes gathered before adaptation. In at least $42 \%$ of these, the changes were appropriate to decrease saccade amplitude based on our current knowledge of cerebellum and brainstem saccade circuitry. Changes in activity during adaptation were not compensating for the potential fatigue associated with performing many saccades. Therefore, many P-cells in the oculomotor vermis exhibit changes in SS activity specific to adapted saccades and therefore appropriate to induce adaptation.
\end{abstract}

\section{Introduction}

A variety of studies suggest that the cerebellum plays an important role in motor learning (Gilbert and Thach, 1977; Optican and Robinson, 1980; Ojakangas and Ebner, 1992; Martin et al., 1996; Barash et al., 1999; Golla et al., 2008; Medina and Lisberger, 2008). Marr (1969) and Albus (1971) proposed an attractive model of cerebellar learning, which implicates the Purkinje (P)cell of the cerebellar cortex as the key element. In their model, climbing fibers, which project from the inferior olive to P-cells, signal the error of dysmetric movements. This error signal is reflected in the probability of occurrence of one of the two types of P-cell action potential, the complex spike (CS) (Soetedjo et al., 2008). Changes of CS activity alter the excitability of P-cells to gradually either decrease, possibly because of long-term depression, or increase because of long-term potentiation. These longterm changes, in turn, alter the activity of the second type of P-cell

Received 0ct. 5, 2009; revised Dec. 22, 2009; accepted Jan. 13, 2010.

This work was supported by National Institutes of Health (NIH) Grants EY00745 and EY019258, and Grant RR00166 from the National Center for Research Resources (NCRR), a component of the NIH. We are grateful for the valuable comments of E. Buzunov, A. Mueller, C. Kaneko, M. Mustari, and J. Phillips on a previous version of this manuscript. The contents of this paper are solely the responsibility of the authors and do not necessarily represent the official views of NCRR or NIH.

Correspondence should be addressed to Albert F. Fuchs, 1959 NE Pacific Street, HSB 1421, Washington National Primate Research Center, Box 357330, University of Washington, Seattle, WA 98195-7330. E-mail: fuchs@u.washington.edu.

D0I:10.1523/JNEUROSCI.4953-09.2010

Copyright $\odot 2010$ the authors $\quad 0270-6474 / 10 / 303715-13 \$ 15.00 / 0$ action potential, the high-frequency simple spikes (SSs), so that their downstream consequences are to reduce movement error.

Saccades are an excellent behavior to test models of neuronal plasticity. First, saccades undergo reversible motor learning (or adaptation) if a target jumps backward as a saccade is made toward it so it appears to overshoot (McLaughlin, 1967) (for review, see Hopp and Fuchs, 2004). Saccades gradually become smaller over hundreds of trials. Second, the subcortical circuitry for saccade generation is well studied (Fig. 1). The superior colliculus (SC) processes signals from several cortical areas and the basal ganglia and generates a motor command to excitatory burst neurons (EBNs) and inhibitory burst neurons (IBNs), which, in turn, drive abducens motoneurons (MNs) to produce a saccade (Moschovakis et al., 1996; Scudder et al., 2002). The SC also sends this saccade motor command to the cerebellum via the nucleus reticularis tegmenti pontis (NRTP), which projects to the oculomotor vermis (OMV) as mossy fibers (Yamada and Noda, 1987). P-cells in the OMV display saccade-related SS activity, which inhibits neurons in the caudal fastigial nucleus (cFN). The cFN projects to contralateral brainstem areas that contain EBNs and IBNs (Noda et al., 1990; Scudder et al., 2000).

In order for adaptation to decrease saccade amplitude, the motoneuron drive to the extraocular muscles must decrease, which can be accomplished by decreasing excitatory input from ipsilateral EBNs and/or increasing inhibitory input from contralateral IBNs (Kojima et al., 2008). These changes of BN activity can be brought about by decreasing and/or increasing the activities of contralateral and/or ipsilateral cFN neurons, respectively 


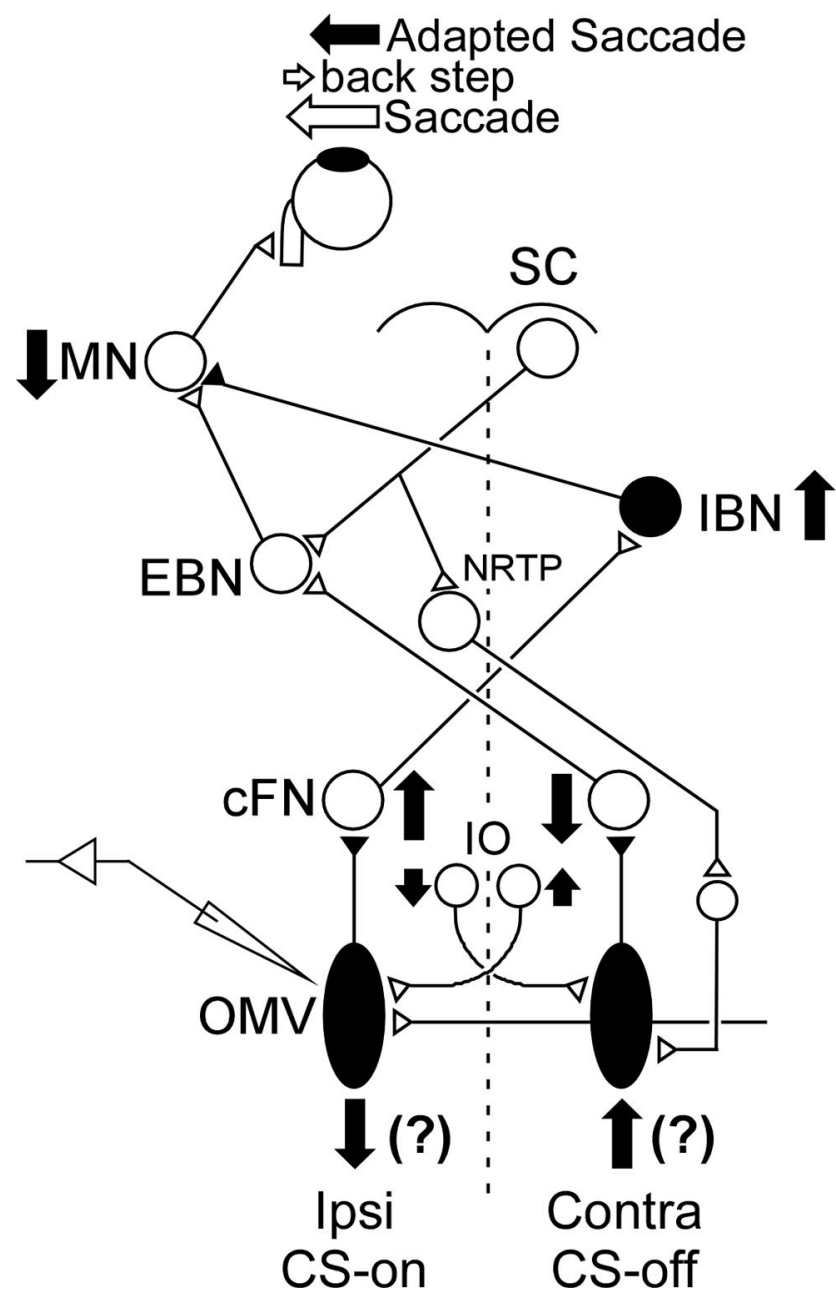

Figure 1. Schematic of the brainstem- cerebellar saccade circuitry underlying an amplitude decrease of leftward saccades. A saccade command from the right SC reaches EBNs, which in turn drive left abducens MNs to generate a saccade. At the same time, a saccade signal from the $\mathrm{SC}$ also reaches both the left and right cerebellar vermis via the NRTP. P-cells in the OMV use this incoming signal to create a saccade-related change in SS activity, which inhibits neurons in the ipsilateral cFN. P-cells also receive a signal from the inferior olive (I0) that creates CSs. Left CFN neurons drive contralateral IBNs, which inhibit left abducens motoneurons. Right CFN neurons project to contralateral EBNs, which excite left abducens motoneurons. The solid vertical arrows indicate the changes in activity that should occur in this saccade circuit to produce smaller leftward saccades during adaptation (left solid arrow). The filled neurons are inhibitory, and the open neurons are excitatory.

(Fig. 1, arrows) (Inaba et al., 2003; Scudder and McGee, 2003). Because P-cells inhibit ipsilateral cFN neurons, SS P-cell activity should increase and/or decrease, respectively, during amplitude decrease adaptation.

We tested this prediction by recording SS activity of single P-cells while we caused amplitude decrease adaptation with intrasaccadic backward target steps. Because the model suggests that changes of SS activity are driven by CS activity, we adapted in the two saccade directions in which the back-step errors produced the highest and lowest CS activity.

\section{Materials and Methods}

Surgical procedures, monkey training, and recording conditions We measured eye movements in three rhesus monkeys (Macaca mulatta; males; $5.0-7.4 \mathrm{~kg}$; monkeys F, B, and W) with the electromagnetic search coil method (Robinson, 1963; Fuchs and Robinson, 1966; Judge et al., 1980). In an aseptic surgery, we implanted a preformed three-turn coil of fine Teflon-coated stainless-steel wire on one eye of each monkey. The ends of the wire exited the orbit through a small hole drilled through the sphenoid bone and ran subcutaneously to a plug affixed to the top of the skull with dental acrylic and titanium screws. This dental acrylic mound also served as one of three lugs that we could grip to prevent head movement. The other two lugs were attached to the skull behind the ears.

After it had recovered from the surgery, each monkey was trained to follow a small visual target with its eyes in a dimly lit booth, while it sat in a primate chair with its head restrained. The targets were an array of light-emitting diodes (LEDs), which were spaced every $1^{\circ}$ along the horizontal and vertical meridians and along the meridians every $45^{\circ}$ in between. Each LED was $61 \mathrm{~cm}$ from the monkey's eyes and subtended $\sim 0.3^{\circ}$, and we illuminated them sequentially to produce target jumps with a maximum excursion of $\pm 20^{\circ}$ from straight ahead. The monkeys were rewarded with applesauce for keeping their eyes within $\pm 3^{\circ}$ windows of the horizontal and vertical components of the illuminated LED for $0.5 \mathrm{~s}$. The on-target criterion was not enforced during the saccadic reaction time. After the monkeys had been trained to fixate an illuminated LED, we trained them to make targeting saccades between successively illuminated LEDs.

After this training was complete, we implanted a recording chamber over a hole in the posterior part of the skull, aimed at the OMV (lobules VIc and VII). The center of the chamber was positioned $14.5 \mathrm{~mm}$ posterior to the interaural axis and directed straight down.

Single-unit neuronal activity was recorded with a home-made Epoxylite-coated tungsten electrode with a tip impedance of $\sim 100$ kohms at $1 \mathrm{kHz}$. The electrode was passed through a guide tube that penetrated the tentorium of the cerebellum. We isolated a unit in the OMV while the monkey was making saccades to track target steps with pseudorandom vectors. We recognized the OMV from the phasic saccade-related bursts in the background activity. We identified an isolated unit as a P-cell if it discharged a CS, which, under our recording conditions, was a positive action potential ending in one or more wavelets that occurred at $1 \mathrm{~ms}$ intervals and was followed by a brief pause in SS activity (for additional details, see Soetedjo et al., 2008). Our data collection concentrated on $\mathrm{P}$-cells that showed a phasic change in SS firing (i.e., a burst, pause, or a combination of the two) associated with targeting saccades in at least one of eight directions spaced every $45^{\circ}$ from 0 to $360^{\circ}$. For example, the P-cell illustrated in Figure $2 \mathrm{~A}$ discharged a small burst of spikes followed by a pause in association with $15^{\circ}$ saccades to the right and down $\left(315^{\circ}\right)$. During such trials, a CS (identified by a large dot) occurred in the error interval between the hypermetric primary saccade and the small corrective saccade. In addition, we examined four cells that did not show phasic changes of SS activity with saccades.

\section{Specific experimental procedures}

Because P-cells often exhibited phasic SS activity for several if not all directions, changes in SS activity during adaptation could occur for saccades in any or all of them. Typically, from 500 to 1000 saccade adaptation trials are required to produce robust amplitude changes in a particular direction. Therefore, if we had attempted adaptation to errors in all eight directions, it would have required between 4000 and 8000 trials during which time $(\sim 1-2 \mathrm{~h})$ we would have had to maintain unit isolation. In our experience, this rarely is possible. We reasoned, therefore, that if changes in CS activity drive adaptation as we hypothesize, our best chance to reveal concomitant changes in SS activity would be along the axis where the change in CS firing probability is greatest. Therefore, for each P-cell, we first determined the preferred (CS-on) direction, took the opposite direction as the CS-off direction, and adapted the monkey only along that axis. We ignored those P-cells whose CSs were not tuned for direction (Soetedjo et al., 2008).

To determine the CS-on direction, we created an error of $\sim 5^{\circ}$ in each of the eight directions by stepping the target backward (toward its initial position) by $5^{\circ}$ during a saccade so the eyes overshot the target. Saccades occurred in blocks of two trials. The first occurred to a centrifugal target jump in one of eight pseudorandom directions from straight ahead. The target jumped to either 12 or $15^{\circ}$, depending on the performance of the individual monkey. When the vector eye velocity of the targeting saccade exceeded $75^{\circ} / \mathrm{s}$, the computer moved the target to either a 7 or $10^{\circ}$ eccentricity, which served as the starting location of the second trial. The next 


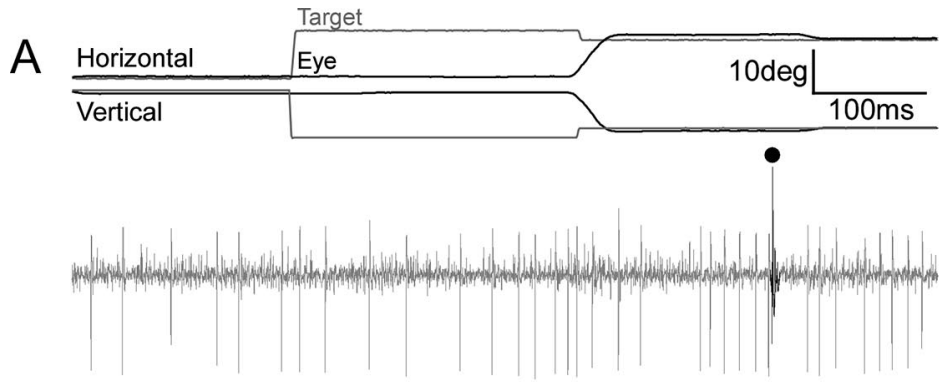

B
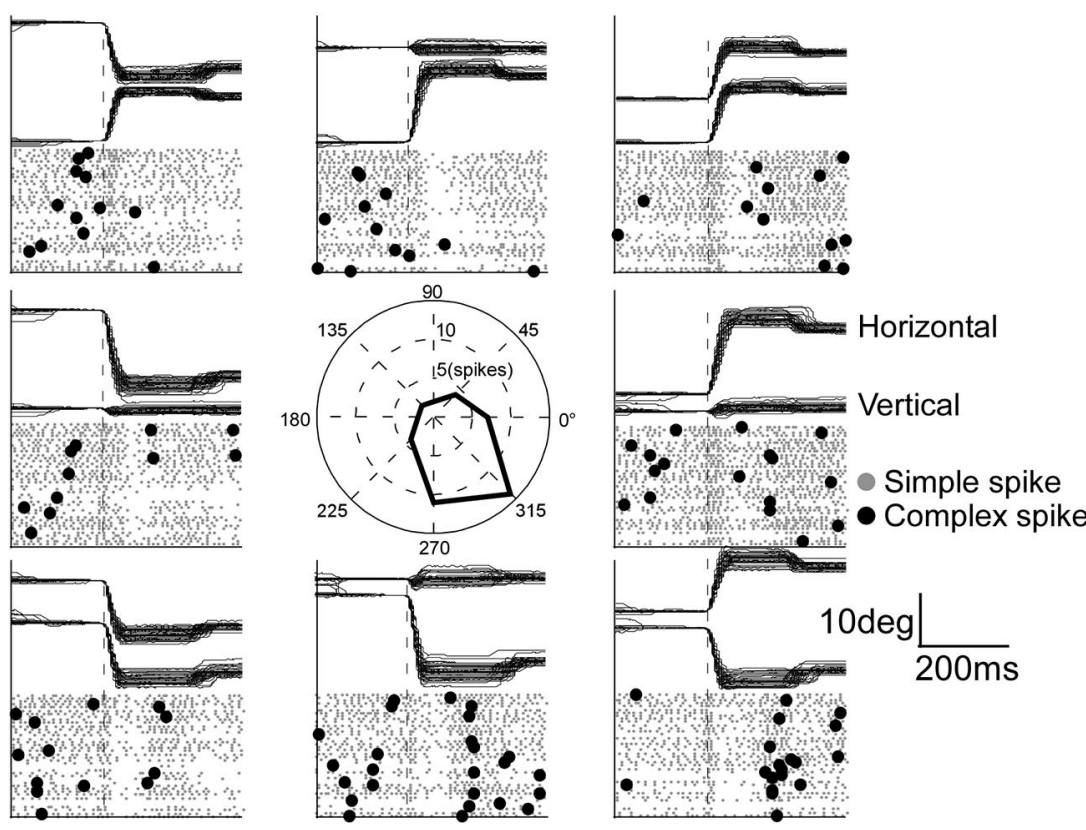

Figure 2. Identification of a P-cell with saccade-related SS activity and determination of its preferred CS direction. $\boldsymbol{A}$, This P-cell in the right vermis discharged a burst then pause of simple spikes (gray action potentials) for a saccade to the right and down (black traces). In this trial, the target (gray traces) jumped to the left and up during the saccade and a single CS (black action potential) occurred while there was an error between the initial and corrective saccade. B, Direction sensitivity of SS activity (gray dots) for saccades and (S activity (black dots) for target errors in the eight different angular directions shown in the center panel. The center panel shows the number of $C S$ s between the primary and corrective saccade for 40 trials in each direction. Most $C S s$ occurred after erroneous saccades to the right and down (see direction tuning curve).

target step brought the target to a $-5^{\circ}$ eccentricity, and the $5^{\circ}$ backward step returned the target to the central location, where the next block of two trials started. We collected at least 50 trials in each direction for monkeys $\mathrm{F}$ and $\mathrm{B}$ and 30 trials for monkey $\mathrm{W}$, who was a poorer worker. For each direction, we displayed the raw unit data on a monitor. Figure $2 B$ displays the raster plot of the SSs (gray) and CSs (black) for 40 trials aligned on saccade onset for a representative P-cell (that in Fig. $2 A$ ). In some directions, the SS activity showed a weak burst near saccade onset (dashed line) followed by variable pause. We counted the number of CSs in the error interval between the erroneous initial saccade and the corrective saccade in each of the eight directions and plotted a tuning curve for CS occurrence (central inset). From such curves generated on-line, we determined the CS-on direction (here right and down) and the CS-off direction (left and up). Our subsequent quantitative off-line analysis (Soetedjo et al., 2008) indicated that the actual CS-on direction always was within $\pm 45^{\circ}$ of the on-line determination. Because backward steps after saccades of the same amplitude and direction were repeated a maximum of 50 times, little, if any, saccade adaptation (reduction of saccade error) occurred while we were determining the preferred CS error direction. Furthermore, while we counted the CSs, we required the monkey to make saccades in all eight directions without backward target steps to erase any adaptation that might have occurred.

After we had identified the direction of primary saccades associated with the highest probability of CS occurrence, we collected SS preadap- tation data for saccades to different target step sizes along the CS-on/off direction axis. During training, we had adapted each animal in each of the eight directions with the back steps that would be used during the recording sessions and from these determined the range of amplitude changes that adaptation would be expected to produce. We selected the sizes of the preadaptation target steps to elicit a range of saccades that would span those behavioral changes. These included the target step that would be used to produce adaptation and target steps that decreased saccade size in $2-3^{\circ}$ increments. In addition to using smaller initial target steps during adaptation $\left(12\right.$ and $\left.15^{\circ}\right)$, we also tested some P-cells using larger initial target steps $\left(20\right.$ and $\left.25^{\circ}\right)$. Use of the larger target steps was an attempt to have adaptation produce a larger absolute change in saccade amplitude to optimize our chances of demonstrating changes in SS activity. We collected $\sim 20$ saccades for each target step size in both the CS-on and -off directions. These preadaptation data allowed us to determine whether any changes in SS activity associated with decreasing amplitudes during adaptation were specific to adaptation or simply attributable to an underlying relationship between SS activity and saccade size.

After we collected the preadaptation data, we induced saccade amplitude reduction in the CS-on and -off directions using the McLaughlin (1967) adaptation paradigm. As a monkey made a saccade toward a target, we stepped the target backward by a fixed amount relative to the target so the eye overshot the displaced target, and a later corrective saccade placed the eye on target. The target stepped randomly in the CS-on or -off direction with the constraint that it could not land at a location $>20^{\circ}$ from straight ahead. As adaptation progressed, the animal overshot less, so we increased the size of the backward step to keep the overshooting error size $>2^{\circ}$. The backward step size started at 8 and $11^{\circ}$ for 20 and $25^{\circ}$ target steps, respectively. For both 12 and $15^{\circ}$ target steps, the size of the backward step started at $5^{\circ}$. We used data only from those units whose SSs were still clearly isolated after there had been a significant decrease in saccade amplitude of $>10 \%$.

\section{Data analysis}

General procedures. We digitized eye and target position signals at $1 \mathrm{kHz}$ and sampled unit activity at $50 \mathrm{kHz}$ using Power 1401 data acquisition/ controller hardware (Cambridge Electronic Design). These data were saved to a hard disk for later analysis. During the experiment, we used a custom program running in Spike2 (CED) to both control the behavior using the Power 1401 and to calculate and display some features of the data on-line (e.g., rasters to determine CS tuning) (Fig. 2).

The saved data were analyzed using custom programs that also ran in Spike2. The program used a velocity criterion of $75^{\circ} /$ s to identify each primary saccade that occurred within $50-800 \mathrm{~ms}$ after the initial target movement. Saccade onset and end were identified by an eye vector velocity criterion of $20 \%$ s. The program also calculated both saccade metrics and reaction time, as well as target amplitude for each trial. Each SS and CS was detected by a trigger threshold and then saved as separate event markers; the marked events were checked by eye. These attributes along with eye and target positions and spike events were exported to MATLAB (The MathWorks) to analyze the relationships between various features of a saccade and the associated SS firing 
A

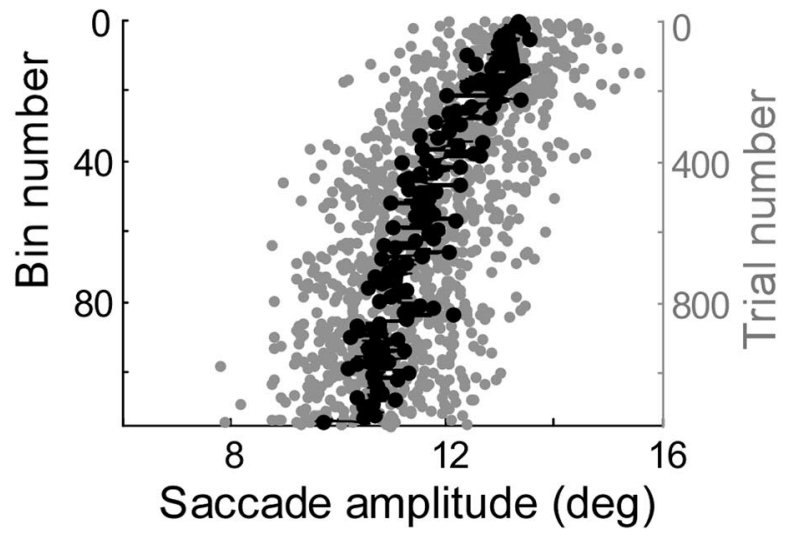

B

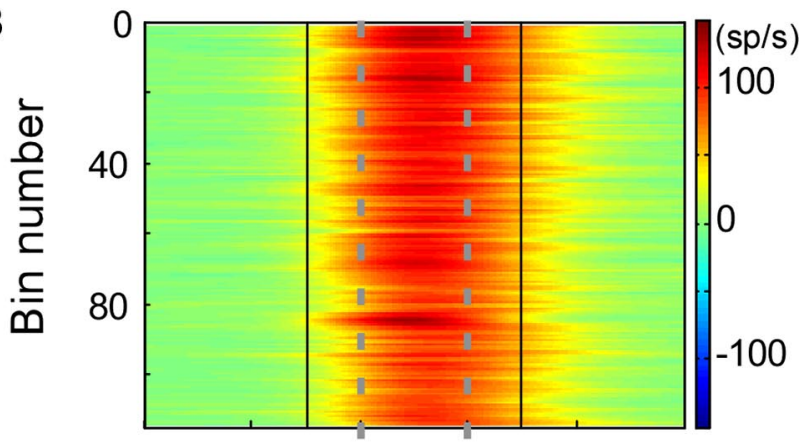

C

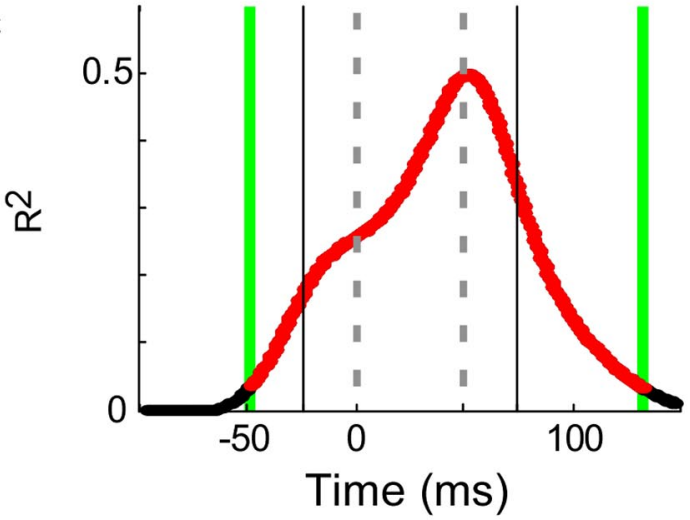

D

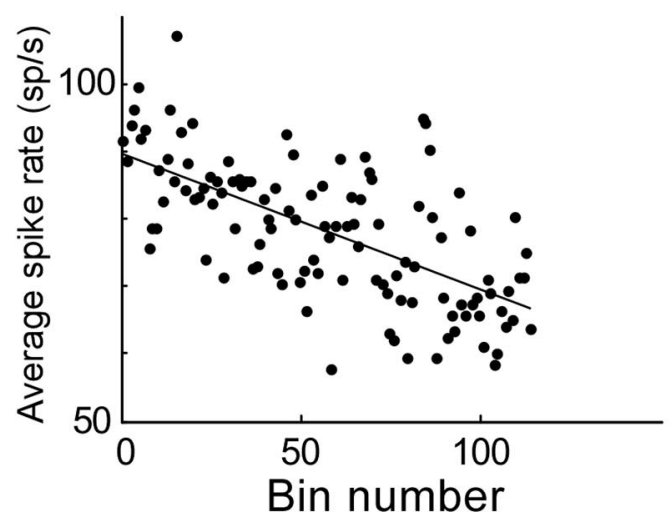

Figure 3. Illustration of the analyses used to document changes in $\mathrm{SS}$ activity during adaptation. $A$, Decrease in saccade amplitude during adaptation. Saccades are shown from top to bottom in order of their occurrence during adaptation (rightward scale). The gray dots show individual saccades; the black dots are averages of every 10 saccades (one bin). B, Average SS SDFs associated with each 10 -saccade bin in $A$ and aligned on saccade onset $(t=0)$. The activity above and below resting rate (green) is indicated by hotter and colder colors, respectively. The gray dashed lines indicate the average saccade duration during adaptation. C, Coefficient of determination $\left(R^{2}\right)$ between SS activity and bin number for every millisecond slice in the trial. The linear regression was significant $(p<0.05)$ for every time slice in the red interval between pattern. We eliminated those saccades that differed in direction from the target vector by $\geq 10^{\circ}$ or whose initial horizontal and vertical eye positions differed from those of the initial target positions by $\geq 1^{\circ}$. These criteria eliminated $<3 \%$ of the trials.

To analyze SS activity, we created a spike density function (SDF) for each trial from $-200 \mathrm{~ms}$ before to $300 \mathrm{~ms}$ after the onset of the saccade by replacing each SS with a Gaussian function (20 ms SD) centered on the spike (Soetedjo et al., 2002). An average rate calculated from an interval between -200 to $-101 \mathrm{~ms}$ was subtracted from the SS activity to equalize the resting rates among trials.

Relationship of simple spike rate with trial number. After we had determined the SS activity for each trial, we measured whether the SS activity changed as adaptations progressed in both the CS-on and -off directions. Figure 3 illustrates these calculations in one CS direction for a representative P-cell. In this example, adaptation reduced saccade amplitude gradually over $\sim 1100$ trials (Fig. $3 A$ ). To view the course of SS changes during adaptation, we calculated the average SS activity over 10 consecutive trials, which reduced the variability of SS responses from trial to trial (Ohtsuka and Noda, 1995; Medina and Lisberger, 2008). The SS activity of each bin is represented by a colorized line (Fig. $3 B$ ), in which the activity above and below resting (green) is indicated by hotter and colder colors, respectively. We stacked the SS activity from top to bottom in the order in which the adaptation trial occurred and aligned all trials with the start of the saccade $(t=0)$ (Fig. 3B). We then tested whether the SS activity changed over the progression of adaptation by calculating the coefficient of determination $\left(R^{2}\right)$ between the binned average spike rate and the sequential bin number (trial number/10; in this example ranging from 1 to 114$). R^{2}$ was calculated at every millisecond (vertical) time slice of the binned SS (Fig. 3B) in the interval between -100 and $150 \mathrm{~ms}$ to produce the plot shown in Figure $3 C$. The time interval in which the SSs showed a significant change $(p<0.05)$ over the progression of adaptation is highlighted in red, -49 to $132 \mathrm{~ms}$ in this example. Because this analysis detects magnitude changes, it also is sensitive to pure shifts in phasic activity, which would cause a change in the binned SS activity at one or more $1 \mathrm{~ms}$ time slices (e.g., the shift of burst end as the adaptation progressed in Figure $3 B$ ). For this P-cell, decreases of both average burst rate and burst duration contributed to the overall width of the significant change of SS activity during the adaptation. We chose this analysis, which detects changes that could be attributable to alterations in either the magnitude or timing of the response, because either change would produce the appropriate downstream effects to validate the model in Figure 1.

To identify an interval of the SS activity to analyze, we determined the average saccade duration throughout adaptation (Fig. $3 B, C$, dashed lines) and analyzed any significant changes that started $25 \mathrm{~ms}$ before and ended $25 \mathrm{~ms}$ after the average duration. Our analysis interval included $25 \mathrm{~ms}$ after the saccade because P-cells inhibit cFN neurons, which often have activity that continues beyond saccade end [Fuchs et al. (1993), their Fig. 2] (Ohtsuka and Noda, 1991; Inaba et al., 2003; Scudder and McGee, 2003). Even if the $R^{2}$ function showed a significant change before and/or after (Fig. $3 C$, green vertical lines) the analysis interval (Fig. 3C, black vertical lines), we considered only the SS activity within the analysis interval for additional analysis. However, if the $R^{2}$ function showed a significant change within only a part of the analysis interval (see Fig. $11 \mathrm{~B}$ ), we limited the additional analysis to just that part.

To determine whether the magnitude of SS activity increased or decreased during adaptation, we calculated the average spike rate within the analysis interval of the binned SS data [i.e., (the area of the SDF within the analysis interval)/analysis interval]. The sign of the correlation coefficient of the linear regression between average spike rate and the se-

$\leftarrow$

the green lines ( $t=-49$ to $132 \mathrm{~ms}$ ). $\boldsymbol{D}$, Average spike rate as a function of bin number (114 bins for this neuron). Average spike rate is the average $\mathrm{SS}$ activity associated with every 10 saccades divided by the analysis interval ( $\pm 25 \mathrm{~ms}$ of the average saccade duration, between vertical black lines in C). Average spike rate decreased significantly with bin number $(p<0.05$; $r=-0.65)$. 

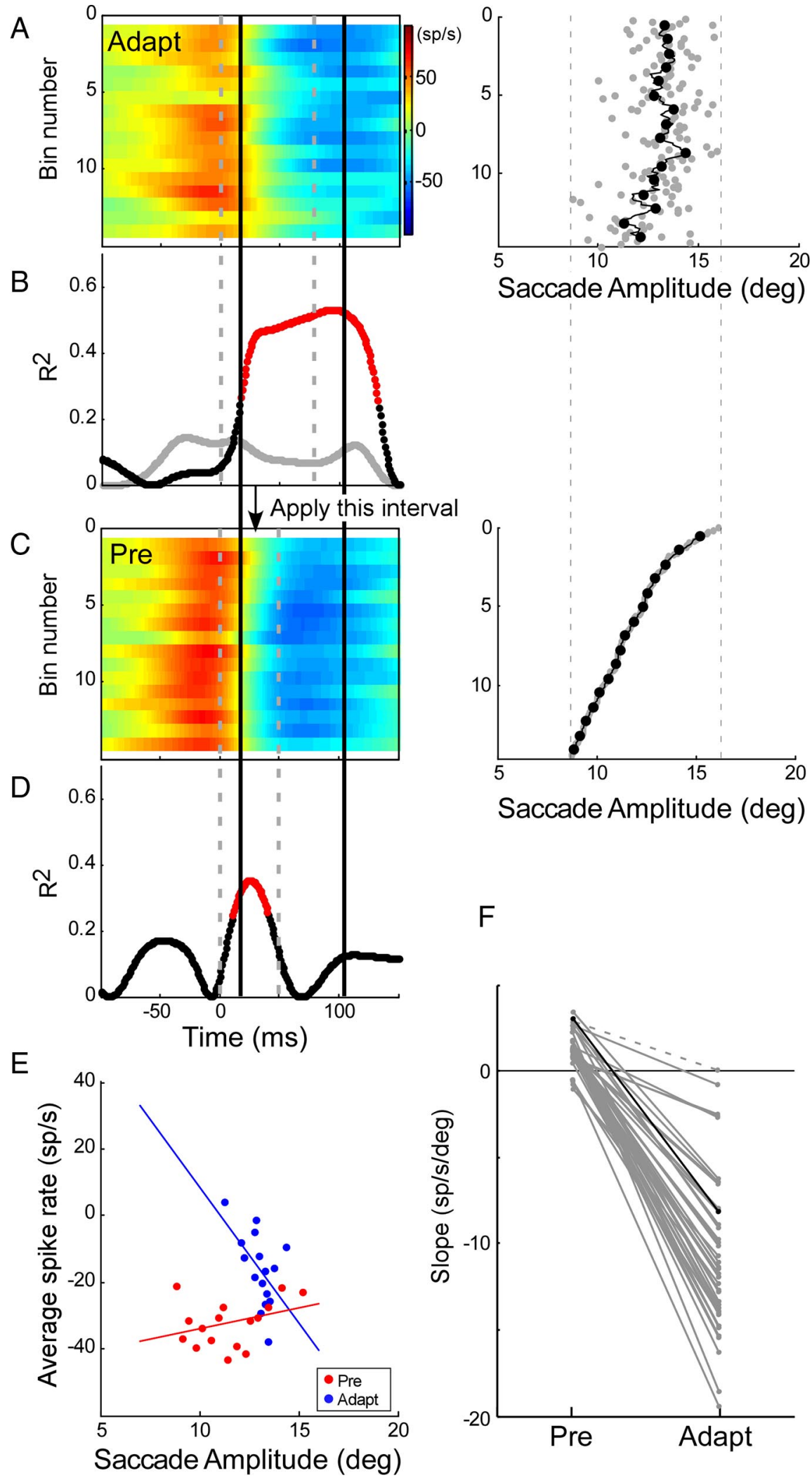

Figure 4. Illustration of the analysis used to compare changes of SS activity with saccade amplitude during adaptation and preadaptation. $\boldsymbol{A}$, SS activity of a resampled adaptation data subset (112 samples from 274 total). $\boldsymbol{C}$, Preadaptation data. Colorized SS activity ordered from top according to binned trial number $(\boldsymbol{A})$ or binned amplitude $(\boldsymbol{C})$; the right panels in $\boldsymbol{A}$ and $\boldsymbol{C}$ show associated changes in saccade amplitude. $\boldsymbol{B}, R^{2}$ of the relationship between SS activity of the adaptation subset in $\boldsymbol{A}$ and bin number; the gray curve shows another adaptation subset with no significant interval. $D, R^{2}$ of the relationship between SS activity in $\boldsymbol{C}$ (left panel) and binned amplitudes in $\boldsymbol{C}$ (right panel). $\boldsymbol{E}$, Average spike rate versus average saccade amplitude for the resampled subset of adaptation data in $\boldsymbol{A}$ (blue dots; $p<0.05 ; r=-0.54$; slope, -8.23 ) and the preadaptation data in $\boldsymbol{C}$ (red dots; $p<0.05$; quential bin number (Fig. 3D) indicated the direction of the change in magnitude of SS activity during the adaptation (in this example: $r=-0.65, p<0.05)$.

Relationship of SS activity with saccade size. Finally, we assessed whether changes in SS activity were specific to adaptation or simply reflected an underlying relationship of SS activity with saccade amplitude. To do so, we compared the relationship between SS activity and saccade amplitude (the saccade amplitude sensitivity) during adaptation with the relationship before adaptation had begun. The range of saccade sizes produced during adaptation was less than that collected before adaptation. Therefore, we first limited the range of preadaptation saccades to the range of sizes of the adapted saccades (Fig. $4 A, C$, right panels, vertical dashed lines). Also, the number of saccades collected during adaptation always was much greater than those taken before adaptation. Therefore, we randomly resampled the more numerous adaptation data to obtain 50 distinct subsets, each with the same number of trials as the preadaptation data that had been adjusted to match the ranges of saccade amplitudes. Each subset was sampled without replacement from the adaptation data, and then the subset was replaced before sampling the next subset. With an equal number of trials, the $p$ values from the analyses of the preadaptation and adaptation data could be fairly compared.

Figure 4 illustrates our analysis in the CS-off direction for a sample P-cell. Figure $4 \mathrm{~A}$ shows the binned SS (left panel) and associated binned amplitude data (right panel) of one of the 50 subsets, which consists of 146 trials randomly resampled from the entire adaptation data set. From SS plots like that in Figure 4A, we determined an analysis interval in the usual way (between black vertical lines, from 17 to $104 \mathrm{~ms}$ ) and applied that interval to the preadaptation binned SS data (Fig. 4C, left panel). The preadaptation data were sorted according to decreasing saccade amplitude (Fig. $4 C$, right panel, top to bottom). Because the number of

$\leftarrow$

$r=0.60$; slope, $\left.3.51 \mathrm{sp} \cdot \mathrm{s}^{-1} \cdot \mathrm{deg}^{-1}\right)$. The dashed lines in the right panels of $\boldsymbol{A}$ and $\boldsymbol{C}$ show that the adapt and pre data have the same amplitude ranges. The smallest adapt averages (black dots) never $g 0<10^{\circ}$ because it is attributable to saccades lying mostly between 11 and $12^{\circ}$. The black dots are the same amplitude averages that appear in $\boldsymbol{E}$. $\boldsymbol{F}$, Slopes of the average spike rate versus amplitude relationships for each of the 50 resampled adaptation subsets and the associated preadaptation data connected by gray lines (black line from data in $\boldsymbol{E}$ ). In seven resampled adaptation subsets, there was no significant interval, so the adaptation slope is plotted as 0 (right point of dashed line). Note that the slopes for the preadaptation regressions differed because the analysis interval varied (for details, see Materials and Methods). For 40 of the 50 subsets, the slopes of preadaptation and adaptation regressions were significantly different. The slopes obtained from adapted saccade data were significantly smaller than those from preadaptation saccade data ( $\chi^{2}$ test; $p<0.05$; mean adapt - pre slopes, -8.27$)$. 
preadaptation trials differed for different P-cells, we first grouped all preadaptation and their paired adaptation data sets into 15 amplitude and SS bins containing approximately equal numbers of trials. We then plotted the average spike rate within the analysis interval (Fig. $4 A-D$, vertical thick lines) against saccade amplitude for both the adaptation data subset (Fig. $4 E$, blue dots) and the preadaptation data (red dots). In this example, the average spike rate of the adaptation data subset showed a significant negative correlation with saccade amplitude $(r=-0.54$; slope, $-8.23 \mathrm{sp} \cdot \mathrm{s}^{-1} \cdot \mathrm{deg}^{-1} ; p<0.05$, blue line). The preadaptation data also were significantly correlated with amplitude, but the correlation was positive $\left(r=0.60\right.$; slope, $3.51 \mathrm{sp} \cdot \mathrm{s}^{-1} \cdot \mathrm{deg}{ }^{-1} ; p<0.05$, blue line).

Figure $4 F$ shows the slopes of all 50 pairs linked by gray lines (the black dots and connecting line are from the data in Fig. $4 E$ ). This figure also shows several variations of this analysis depending on whether or not the analysis interval could be determined from the adaptation data subset. When we attempted the analysis on the 50 adaptation data subsets for this unit, analysis intervals could be determined for only 43 . For the remaining seven, $R^{2}$ never reached significance (an exemplar subset is illustrated by the gray curve in Fig. $4 B$ ). For such subsets, we considered the slope of the relationship between average spike rate and amplitude to be zero. However, the SS activity associated with preadaptation saccades did show a relationship with binned saccade amplitude. The $R^{2}$ associated with that relationship reached significance between 11 and $40 \mathrm{~ms}$ (Fig. $4 D$, entire redlined section; note that all preadaptation data are the same, i.e., those in Fig. 4C). Within this interval, the slope of the average spike rate versus preadaptation saccade amplitude relationship was 3.31 $\mathrm{sp} \cdot \mathrm{s}^{-1} \cdot \mathrm{deg}{ }^{-1}$. This slope of the preadaptation data with the zero slope for the adaptation data produced the dashed line in Figure $4 F$ (represents seven subsets). Of all 50 subsets, 40 had slopes of the saccade amplitude sensitivity during adaptation that were significantly different from those before adaptation based on both their confidence intervals. Note that, although the preadaptation data were always the same, the slope of the average spike rate versus amplitude relationship could differ because individual analysis intervals were determined on different subsets of resampled adaptation data. These 40 subsets include the 7 for which the significant analysis interval could be identified only in the preadaptation data. The last possibility is that a significant analysis interval could not be identified in either the adaptation subset or the preadaptation data. In this case, we took the slopes of both data sets to be zero. The exemplar unit in Figure 4 had no such cases.

Finally, we used a $\chi^{2}$ test to determine whether the number of adaptation data subsets with slopes that differed significantly from the preadaptation slopes was significantly larger than the number that would occur by chance. For 50 subsets, 35 or more subsets with significantly different slopes are required to achieve significance $(p<0.05)$. Because the P-cell illustrated in Figure 4 had 40 subsets that had significantly different slopes, we could conclude that, for this P-cell, the relationship of firing rate with amplitude during adaptation was significantly different from that before adaptation.

The mean difference between all 50 adaptation and preadaptation slopes was $-8.27 \mathrm{sp} \cdot \mathrm{s}^{-1} \cdot \mathrm{deg}^{-1}$. Therefore, in this example, when saccade amplitude decreased during adaptation, the SS firing rate increased more than it would have for similar decreases during preadaptation (i.e., for saccades not associated with adaptation). We consider the difference of the slopes between the adaptation and preadaptation data as the signal that is unique to adaptation (i.e., a functional adaptation signal). If the $\chi^{2}$ test of those 50 data subsets failed to achieve significance, we concluded that they were statistically indistinguishable from the slopes of the preadaptation data. For P-cells with such subsets, we considered their mean slope differences to be zero.

Our data processing is admittedly rather complicated. However, the multiple steps in our analysis were necessitated by the variability of SS discharges, either within a single P-cell or across different P-cells, and the limited number of preadaptation trials. In addition, the random resampling method also had the advantage of providing an estimate of the variability of the slopes (Fig. $4 F$ ), which lent more credibility to our statistical test.

These experiments were performed in accordance with the Guide for the Care and Use of Laboratory Animals (1997) and exceeded the minimal

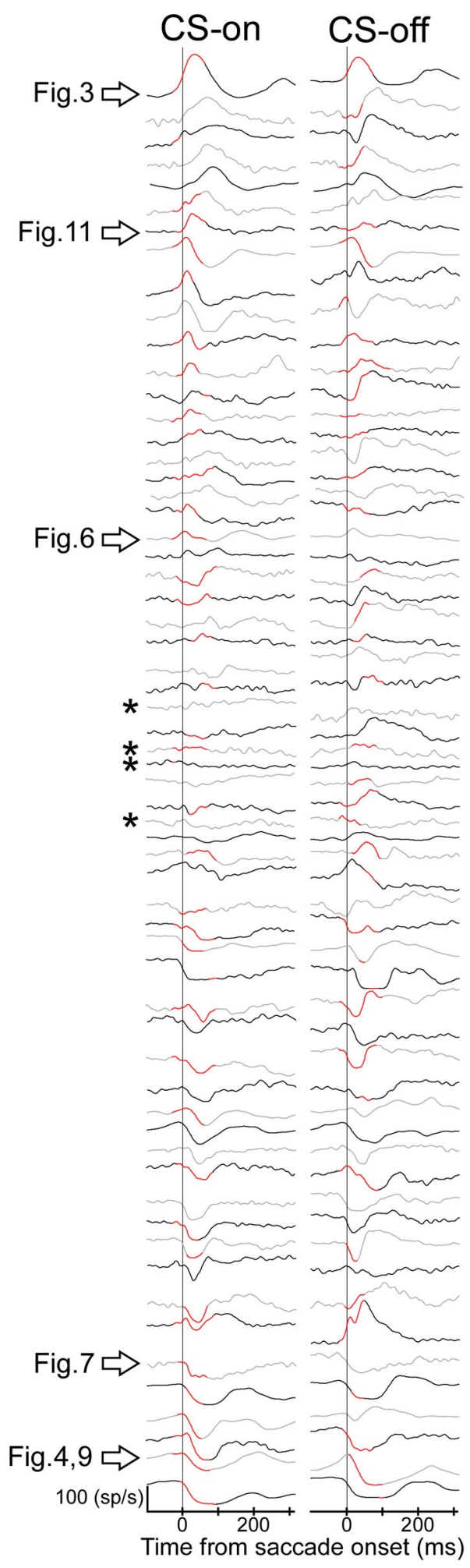

Figure 5. Average SS discharge patterns of all $61 \mathrm{P}$-cells for preadapted saccades in the CS-on and -off directions. All patterns are aligned on saccade onset. We loosely ordered the activity from top to bottom according to whether it exhibited a burst, first a burst and then a pause, little perisaccadic change (identified by 4 asterisks), or a pause in the (S-on direction. The arrows identify the P-cells illustrated in other figures. To make it easier to compare CS-on and -off direction activity for the same unit, alternate units are identified by gray and black lines. For the different neurons, saccades could be in response to targets of $12,15,20$, or $25^{\circ}$. The red highlighted regions on the SDFs indicate the analysis intervals used for each cell and direction. The SDFs without red highlighted regions had no significant analysis interval. 
CS-on

A

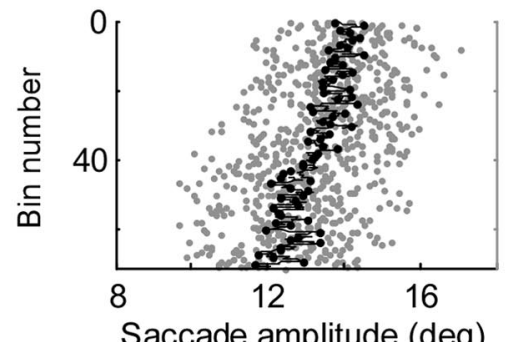

B

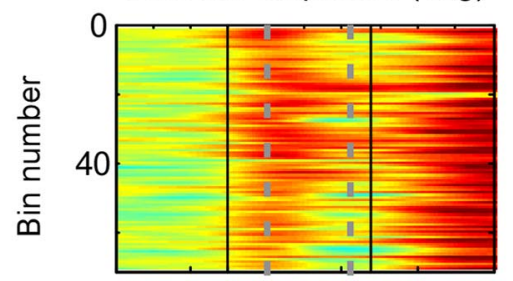

C
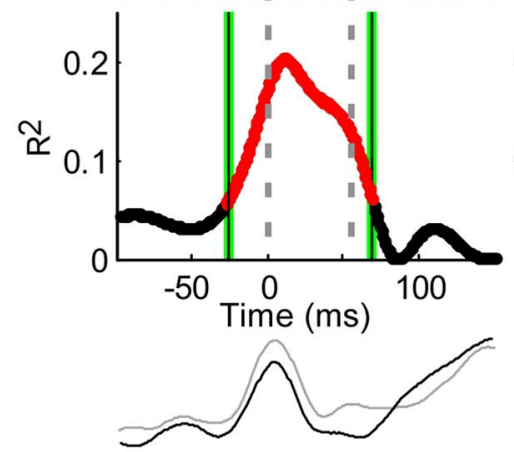

D

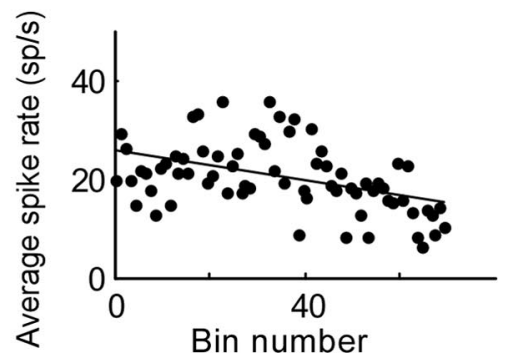

CS-off
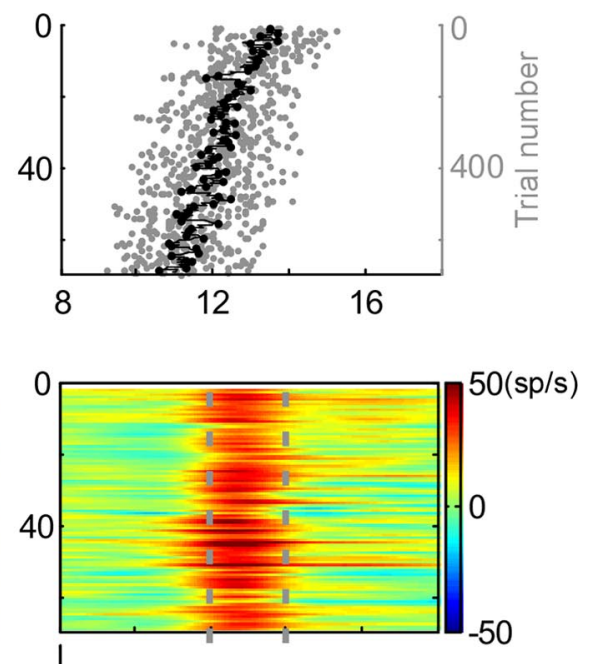

$15^{\circ}$, and the mean percentage amplitude reduction was $14.3 \pm 3.2 \%$ in the CS-on direction and $15.5 \pm 6.3 \%$ in the CS-off direction; the amplitude reductions in the two directions were not significantly different $(t$ test, $p=0.22)$. For the remaining 25 P-cells, the initial target step was either 20 or $25^{\circ}$, and the mean amplitude reduction was $17.1 \pm 3.7$ and $18.1 \pm$ $3.7 \%$ in the CS-on and -off directions, respectively; there was no significant difference between the percentage amplitude reductions in the two directions $(p=0.11)$.

Phasic simple spike activity for saccades As shown in previous studies (Ohtsuka and Noda, 1995; Soetedjo and Fuchs, 2006; Soetedjo et al., 2008), P-cells in the oculomotor vermis exhibit a variety of saccade-related SS discharge patterns. Figure 5 shows the average SS activity associated with preadapted saccades to target steps in the CS-on and -off directions aligned on saccade onset for all $61 \mathrm{P}$-cells. For ease of viewing, we sorted by eye the perisaccadic SS activity in the CS-on direction according to whether it exhibited a burst (top), little phasic activity (middle), or a pause (bottom). Clearly, there are a variety of phasic SS patterns accompanying CS-on direction saccades, and some P-cells exhibit different patterns in the CS-on and -off directions. Not only are the qualitative patterns quite variable, but their quantitative details (e.g., the magnitude, timing, and duration of the phasic activity) are as well. Moreover, as seen in Figure $2 B$, even for the same P-cell, SS firing patterns accompanying very similar saccades can be quite variable. The red highlighted region indicated the analysis interval for each P-cell. Those analysis intervals varied from unit to unit. Finally, four cells (identified by asterisks) exhibited little, if any, phasic SS activity for either saccade direction.

Relationship of simple spike activity to saccade amplitude during adaptation The change of activity of a P-cell with a saccade-related SS burst during adaptarequirements recommended by the Institute of Laboratory Animal Resources and the Association for Assessment and Accreditation of Laboratory Animal Care International. All the procedures were evaluated and approved by the local Animal Care and Use Committee of the University of Washington.

\section{Results}

We recorded the activity of $61 \mathrm{P}$-cells in the oculomotor vermis during amplitude reduction adaptation in three monkeys. For all $61 \mathrm{P}$-cells, the amplitude of the last 20 saccades of adaptation was significantly smaller than that of the first 20 by $>10 \%(p<0.05$, Student's $t$ test). For $36 \mathrm{P}$-cells, the initial target step was either 12 or tion is shown in Figure 6. Before adaptation, this P-cell discharged a burst of SSs for saccades to a $15^{\circ}$ target step in both the CS-on and -off directions (Fig. $6 \mathrm{~B}$, perisaccadic red portions of the SS discharge at the tops of the panels). Adaptation produced by repeated trials with intrasaccadic backward target steps caused a reduction of saccade amplitude in response to a $15^{\circ}$ target step of 2.03 and $2.75^{\circ}$ in the CS-on and -off directions, respectively (Fig. 6A). As adaptation reduced saccade amplitude, the saccaderelated burst of SSs decreased in the CS-on direction but remained unchanged in the CS-off direction (Fig. 6B). In the CS-on direction, the relationship between average burst rate and 
bin number is statistically significant in the interval between -28 and $69 \mathrm{~ms}$ (Fig. $6 C$, reddened portion of curve), but in the CS-off direction, the relationship does not reach significance anywhere in the trial (see Materials and Methods for statistical details). The changes of the burst in the CS-on and -off directions also can be seen by comparing the average SDFs for the first and last 50 saccades during adaptation (Fig. 6C, bottom traces). However, as mentioned in Materials and Methods, this simple comparison is not diagnostic for other P-cells in which the changes during adaptation tend to wax and wane. The average spike rate decreased as adaptation progressed in the CS-on direction $(r=$ -0.45 ) (Fig. 6D). Examination of Figure $6 B$ suggests that the change is attributable mainly to a change in the magnitude rather than the timing of the burst. The increase in firing rate after the saccade is over is unusual; like this neuron, if it does occur, it does not change with adaptation.

An example of the changes undergone by a pausing $\mathrm{P}$-cell during adaptation is shown in Figure 7. Before adaptation, a pause of SS activity occurred in both the CS-on and -off directions (Fig. $7 B$, perisaccadic blue SS discharge at the tops of the panels). During adaptation, the amplitude of saccades to $12^{\circ}$ target steps decreased by $1.9^{\circ}$ and $2.2^{\circ}$ in the CS-on and -off directions, respectively. After adaptation, the pause during the average saccade duration (vertical dashed lines) in the CS-on direction began earlier, but there was little change in the pause during the saccade in the CS-off direction. In the CS-on direction, the relationship between the SS and binned trial number is statistically significant in the interval between -6 and $110 \mathrm{~ms}$ (Fig. $7 B$, reddened part); however, the relationship between the SS activity and binned trial number does not reach significance at any time in the CS-off direction. In the CS-on direction, the correlation of the average spike rate (between black lines) during adaptation had a significant slope of $-0.44(r=-0.61)$ (Fig. $7 C)$.

Figure 8 shows the distribution of correlation coefficients $(r)$ of the linear regressions between average spike rate and bin number for the CS-on and -off directions for all $61 \mathrm{P}$-cells. If no interval showed a significant change (e.g., the CS-off direction for the P-cell in Fig. 7B), we considered that $r$ to be zero. The $r$ values for nonphasic and phasic P-cells were intermingled, so in the following analyses we will consider all P-cells together. Eightythree percent (50 of 61) of all P-cells showed a significant change in average spike rate during adaptation in at least one of the two CS directions. Of the four cells without phasic perisaccadic SS activity before adaptation, two nevertheless underwent changes during adaptation. For our entire population of $61 \mathrm{P}$-cells, 28 showed changes in SS rate in both CS directions, 9 in only the CS-off direction, 13 in only the CS-on direction, and 11 in neither direction (Fig. 8, pie chart).

$B$
CS-on
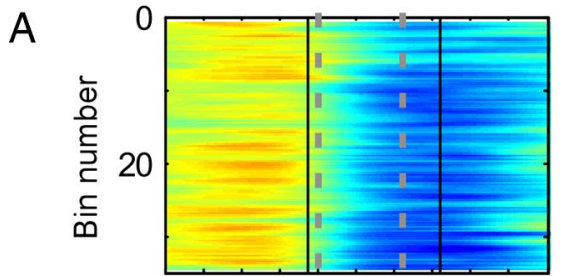

Figure 7. Change of SS activity during adaptation for a pausing P-cell. CS-on and-off directions were 135 and $315^{\circ}$, respectively. $A$, Colorized binned SS activity during adaptation arranged in order of occurrence and aligned on saccade onset. The gray dashed lines indicate average saccade duration during adaptation (CS-on and -off, $54.7 \mathrm{~ms}$ ). $\boldsymbol{B}, R^{2}$ for linear regressions between $S S$ (A) and bin number for every $1 \mathrm{~ms}$ time slice. Correlations were significant (reddened part of curve, green vertical lines) between -6 to $114 \mathrm{~ms}(p<0.05)$ in the CS-on direction. In the CS-off direction, $R^{2}$ did not reach significance at any time. The bottom two traces in $\boldsymbol{B}$ show the average SDFs of the first (gray) and last (black) 50 trials during saccade adaptation. $\boldsymbol{C}$, Average spike rate as a function of bin number for the CS-on direction; the decrease was significant (slope, $-0.44 ; p<0.05 ; r=-0.61$ ).

\section{Comparisons of saccade amplitude sensitivity before and after adaptation}

Because our adaptation paradigm caused a decrease in saccade amplitude, any changes in SS activity with binned trial number might not be unique to adaptation but might simply reflect an underlying relationship of SS activity with saccade amplitude. To evaluate this possibility, we compared the relationship of SS activity with saccade amplitude during adaptation with that for a comparable range of saccades gathered before adaptation had begun.

Figure 9 shows this comparison for a representative P-cell. This $\mathrm{P}$-cell exhibited a pause in SS activity in the CS-on direction and a burst-pause pattern in the CS-off direction during both the binned adaptation (Fig. 9A) and preadaptation trials (Fig. 9B). In the CS-on direction, 18 resampled subsets of adaptation data had no significant analysis interval (Fig. 9C), and the preadaptation data showed no significant relationship with amplitude; thus, these 18 subsets had equal slopes of zero for both adaptation and preadaptation data. Although significant intervals could be identified in the 32 remaining adaptation subsets, only 5 had slopes that were significantly different from those of the preadaptation data. Therefore, in this CS-on direction, the relationship between SS activity and amplitude during and before adaptation was statistically indistinguishable $\left(\chi^{2}\right.$ test, $\left.p>0.05\right)$. For this direction, the "saccade amplitude sensitivity" did not change with adapta- 


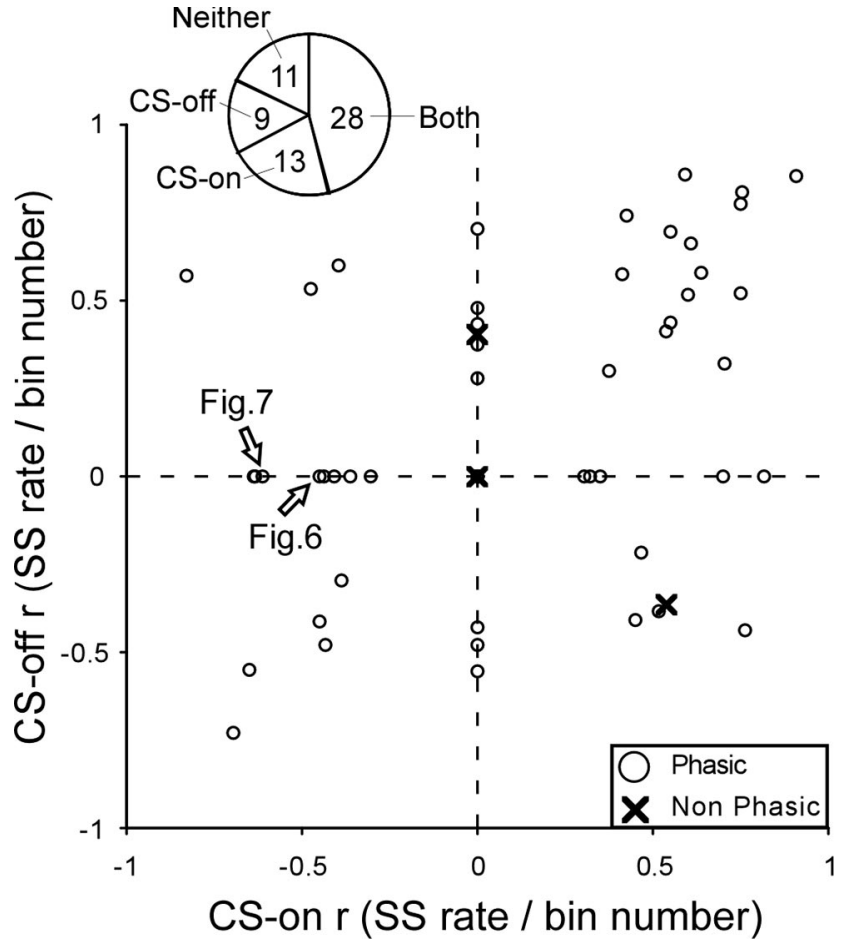

Figure 8. Distribution of correlation coefficients of linear regressions between average spike rate and bin number during adaptation for the CS-off and -on directions for all 61 P-cells. Xs indicate the four P-cells with little phasic activity. The arrows identify the P-cells that appeared in the two previous figures. The pie chart shows the number of units with changes in either the CS-on or -off directions, in both directions or in neither direction.

tion. In the CS-off direction, 40 of 50 adaptation data subsets had slopes that differed significantly from those of preadaptation data. Therefore, the relationship between SS activity and amplitude during and before adaptation was significantly different $\left(\chi^{2}\right.$ test, $p<0.05$ ), indicating that, in this direction, the saccade amplitude sensitivity did change during adaptation.

We now will consider which changes in SS activity with saccade amplitude would be appropriate to cause the changes in firing rates that are predicted for amplitude reduction by the model in Figure 1. First, we suggest that the change in SS activity unique to adaptation is that revealed during adaptation relative to that which occurs for amplitude-matched saccades before adaptation. We call this difference the "functional adaptation signal." Second, we suggest that the reciprocal signal in the two cFNs, which leads to the changes in saccade amplitude illustrated in Figure 1, results from a differential SS signal in the CS-on and -off directions. In the illustrated reduction of leftward saccades in Figure 1, a decrease and increase in SS activity for ipsilateral (CSon) and contralateral (CS-off) P-cells, respectively, would produce the desired changes in $\mathrm{cFN}$ activity (black arrows). Figure $10 \mathrm{~A}$ shows the slopes of the functional adaptation signals in both the CS-off and -on directions for all 61 neurons. A positive value indicates that SS activity decreased more for decreases in saccade amplitude during adaptation than for similar decreases in amplitude before adaptation. A value of 0 indicates that there was no significant difference between adaptation and preadaptation data.

Where on Figure 10 do the units with changes appropriate to cause the amplitude decrease illustrated in Figure 1 lie? That scenario requires a decrease in SS activity in the CS-on direction with decreasing amplitude (i.e., a positive functional adaptation sig- nal) and/or an increase in SS activity the CS-off direction with decreasing saccade amplitude (i.e., a negative functional adaptation signal). Such units lie in the fourth quadrant of Figure $10 \mathrm{~A}$ and on its axes. This includes units with positive slopes in their CS-on directions and either negative or zero slopes in their CS-off directions, and units with zero slopes in their CS-on directions and negative slopes in their CS-off directions. In total, they constitute 16 of all our neurons (Fig. $10 \mathrm{~B}$, fourth quadrant histogram bar). Units with the opposite and therefore functionally inappropriate patterns constitute 13 of our P-cells (Fig. 10A, second quadrant and its axes; Fig. $10 \mathrm{~B}$, second quadrant histogram bar). The remaining two responsive categories include those with either positive (first quadrant histogram bar) or negative (third quadrant histogram bar) functional adaptation signals in both the CS-on and -off directions. Data from the 23 cells located at $(0 / 0)$ in Figure $10 \mathrm{~A}$ showed no slope differences between adapted and preadapted data in either direction ( $0 / 0$ histogram bar). Of these, 17 exhibited significant slopes both during and before adaptation in one or both CS directions, but the two slopes were not significantly different. The remaining six exhibited significant slopes neither during nor before adaptation in both directions. For 38 of our total $61 \mathrm{P}$-cells $(62 \%)$, the functional adaptation signal had a significant slope in either the CS-on or -off directions. Of these, 11 did not exhibit a significant slope during adaptation in at least one direction but did before adaptation.

Finally, the distribution of units in the five histogram bars of Figure $10 \mathrm{~B}$ varied somewhat if the data had been gathered in experiments with small ( 12 and $15^{\circ}$, gray bars) and large (20 and $25^{\circ}$, white bars) target steps. In particular, units in the fourth quadrant bar were clearly much more plentiful when small target steps were used, a point we consider in the Discussion.

In addition to the resampling analysis we performed to obtain the data in Figure $10 \mathrm{~B}$, we also estimated the confidence intervals of the slopes of the adaptation and preadaptation saccade amplitude sensitivities by considering both original data sets. In this approach, the significance of the difference in the two data sets is based on the variance of the actual data. As shown in Figure 10C, this second approach produced similar distribution of P-cells in the different quadrants.

\section{Are changes in SS activity during adaptation related to fatigue?}

To test whether the change in SS activity during adaptation is meant to help compensate for fatigue (Golla et al., 2008), we performed two additional experiments. Before adaptation, we recorded the SS activity of two P-cells while the monkey made the number of saccades he would make in a subsequent adaptation. We assumed that the time course of fatigue generated in these preadaptation and following adaptation trials would be comparable. For the unit illustrated in Figure 11, SS activity in the CS-on direction showed a significant increase over a small interval during the fatigue control trials (Fig. $11 \mathrm{~B}$ ), but during the following adapt trials (Fig. 11A), the significant interval started much earlier and was much broader (Fig. 11C). To compare the fatigue control and adaptation data, we applied the adaptation analysis interval to both data sets, calculated the average spike rate and plotted it against binned trial number. The adaptation data had a significant slope $\left(0.31 \mathrm{sp} \cdot \mathrm{s}^{-1} \cdot \mathrm{bin}^{-1}\right)$, whereas the fatigue data did not (slope, 0.0 ). In the CS-off direction, no significant change of SS activity was detected during the fatigue trials (Fig. $11 E$ ), but a very robust SS change occurred during adaptation (Fig. $11 \mathrm{~F}, \mathrm{G}$ ). For the other P-cell, there was little change in SS activity in the $\mathrm{CS}$-on direction during the fatigue test, but a robust increase 
during adaptation. In the CS-off direction, however, changes during the fatigue test and adaptation were comparable.

For these two cells, the data indicate that the maintenance of saccade amplitude in the face of the possible fatigue associated with the repetition of many saccades (Fig. 11A, trials approximately -300 to 0 ) was not always associated with compensatory changes in SS activity. In contrast, when there was a change in saccade amplitude during adaptation (Fig. $11 C, F$, trials 0 to $\sim 400$ ), there always was a substantial change in SS activity over approximately the same number of saccades.

\section{Discussion}

\section{Major findings}

Of the $61 \mathrm{P}$-cells we studied in the oculomotor vermis, 38 (or 62\%) behaved differently for saccades whose size changed because of adaptation than for similarsized saccades gathered before adaptation (Figs. 9, 10). We consider that the difference in the slopes of the firing rate versus saccade amplitude relationship between adapted and preadaptation saccades reflects a functional adaptation signal that participates in the reduction of saccade size. The functional adaptation signal could be associated with either the CS-on or CS-off directions or both. Functional adaptation signals could occur in P-cells with any of the phasic preadaptation activity patterns with saccades (i.e., either bursts, pauses, or some combination of the two) (Fig. 5), as well as those with little preadaptation saccade-related activity.

The changes in SS activity during adaptation do not appear primarily to compensate for the potential fatigue produced by the numerous trials (Golla et al., 2008). In both P-cells we tested, SS activity always exhibited a robust change (Fig. 11, both CS directions) when a large number of adaptation trials produced substantial decreases in amplitude, but little, if any, change for a comparable number of saccades whose amplitudes remained constant. It is possible, however, that some component of the change in activity during adaptation does compensate for fatigue.

Two characteristics of SS changes during adaptation are noteworthy. For some P-cells (e.g., those illustrated in Figs. 3 and 7), significant changes began before and continued after the saccade ended. Although our $20 \mathrm{~ms}$ Gaussian filter accounts for some of this spread, part of the change in activity beyond saccade end might help modify the timing of the late phasic activity of the target cFN neurons (Fuchs et al., 1993; Scudder and McGee, 2003). Also, for some P-cells, changes in SS discharge occurred relatively smoothly throughout adaptation (Fig. 3), whereas for others (Fig. 6), they did not. This difference is not explained by differences in the changes of adapted saccade amplitude, which, for example, occurred equally smoothly for both sample neurons. Perhaps the synaptic adaptation process is noisier for some cells
CS-on
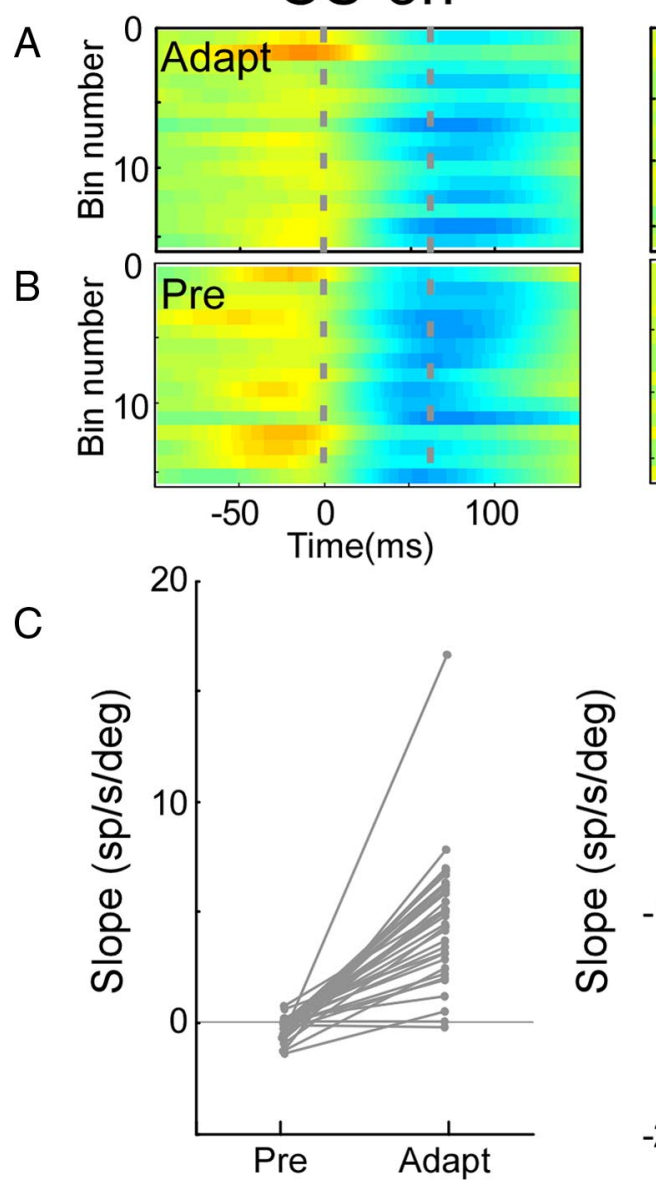

CS-off
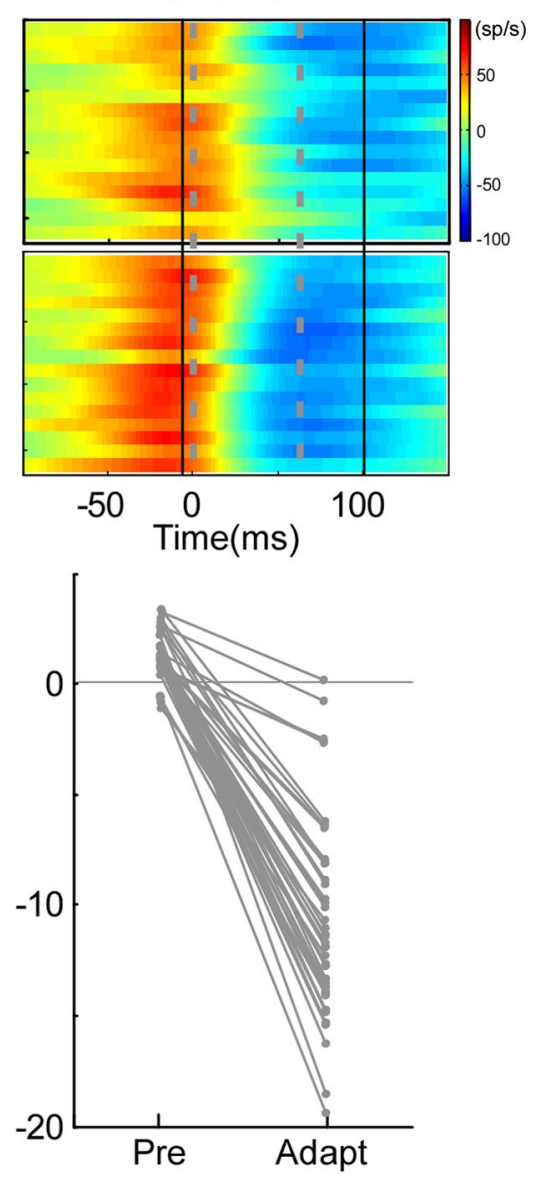

Figure 9. Comparison of the regressions of SS activity with saccade amplitudes during adaptation and preadaptation for a obtained for preadaptation saccades (average of all 50,1.41 \pm 1.11 ) were significantly greater $\left(\chi^{2}\right.$ test; $p<0.05$; mean diff, -8.27 ) than those for adaptation saccades (average of all $50,-6.86 \pm 4.28$ ).

than others, but the total population activity produces a smooth amplitude change. Finally, adaptation data like that in Figure 6 (CS-off), which occur in clusters, make it clear why it is necessary to track SS changes throughout the entire adaptation, rather than simply to compare activity at just the beginning and end.

\section{Relationship of our data to the model proposed in Introduction}

Although $62 \%$ of our P-cells showed different relationships of SS activity with saccade amplitude for adapted than nonadapted saccades, the pattern of the relative change in the CS-on and CS-off direction showed considerable variation (Fig. 10). Nevertheless, based on our current knowledge of the behavior and connectivity of saccade-related neurons in the oculomotor cerebellum and brainstem, some P-cells exhibited discharge patterns that were appropriate to drive a decrease in the size of saccades during adaptation as illustrated in Figure 1. Such patterns include a decrease in SS activity in the CS-on direction [Fig. 1, $\downarrow$ (?)] and/or an increase in SS activity the CS-off direction [Fig. 1, $\uparrow(?)]$. In Figure $10 A$, such cells are found in the fourth quadrant 

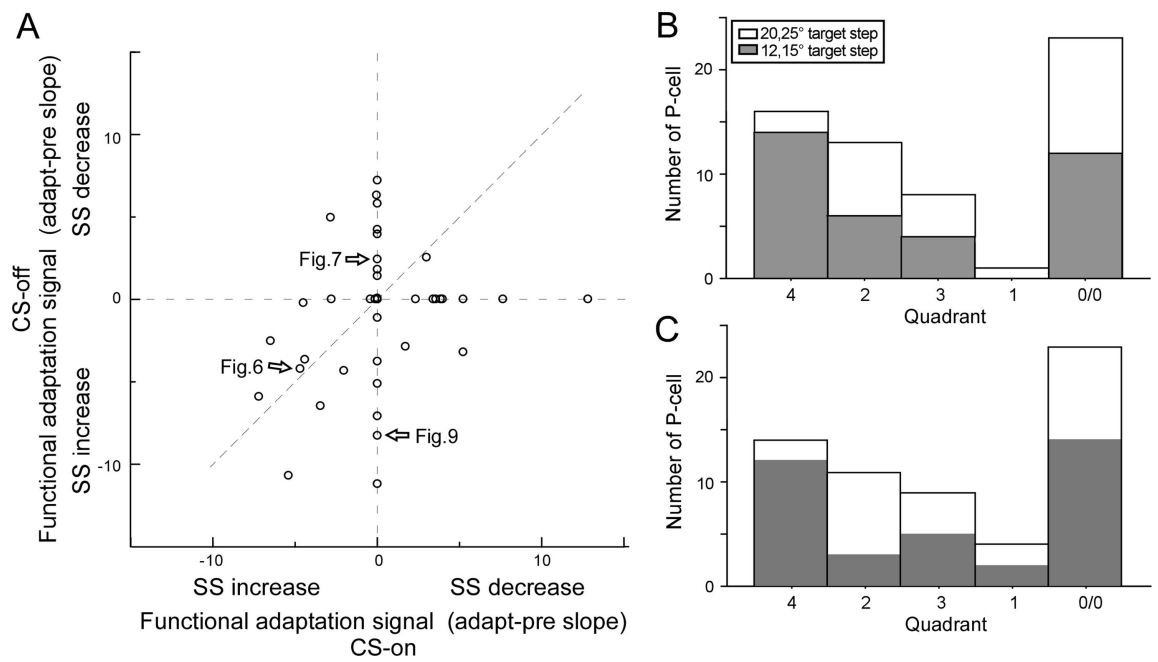

Figure 10. Comparison of the functional adaptation signals in all 61 P-cells. $\boldsymbol{A}$, The functional adaptation signal (mean difference of the slopes of the average spike rate vs amplitude relationship for adapted minus preadaptation saccades) in the CS-off and CS-on directions. Because adaptation caused a decrease in saccade amplitude, negative slopes indicate an increase in SS activity and positive slopes indicate a decrease. The arrows identify P-cells appearing in previous figures. $\boldsymbol{B}$, Number of P-cells whose functional adaptation signals in the CS-on and -off directions $(\boldsymbol{A})$ place them in five different functional categories. Categories include P-cells with data that lie in the fourth quadrant and on its axes (left histogram bar), data that lie in the second quadrant and on its axes (second bar), data from the third quadrant (third bar), data from the first quadrant (fourth bar), and data at the origin (fifth bar). The gray bars indicate that the primary saccade was to 12 or $15^{\circ}$ target steps; the white bars indicate data for primary saccades to 20 or $25^{\circ}$ target steps. C, Units categorized as in $\boldsymbol{B}$ but analyzed on the whole data set without resampling (see text).

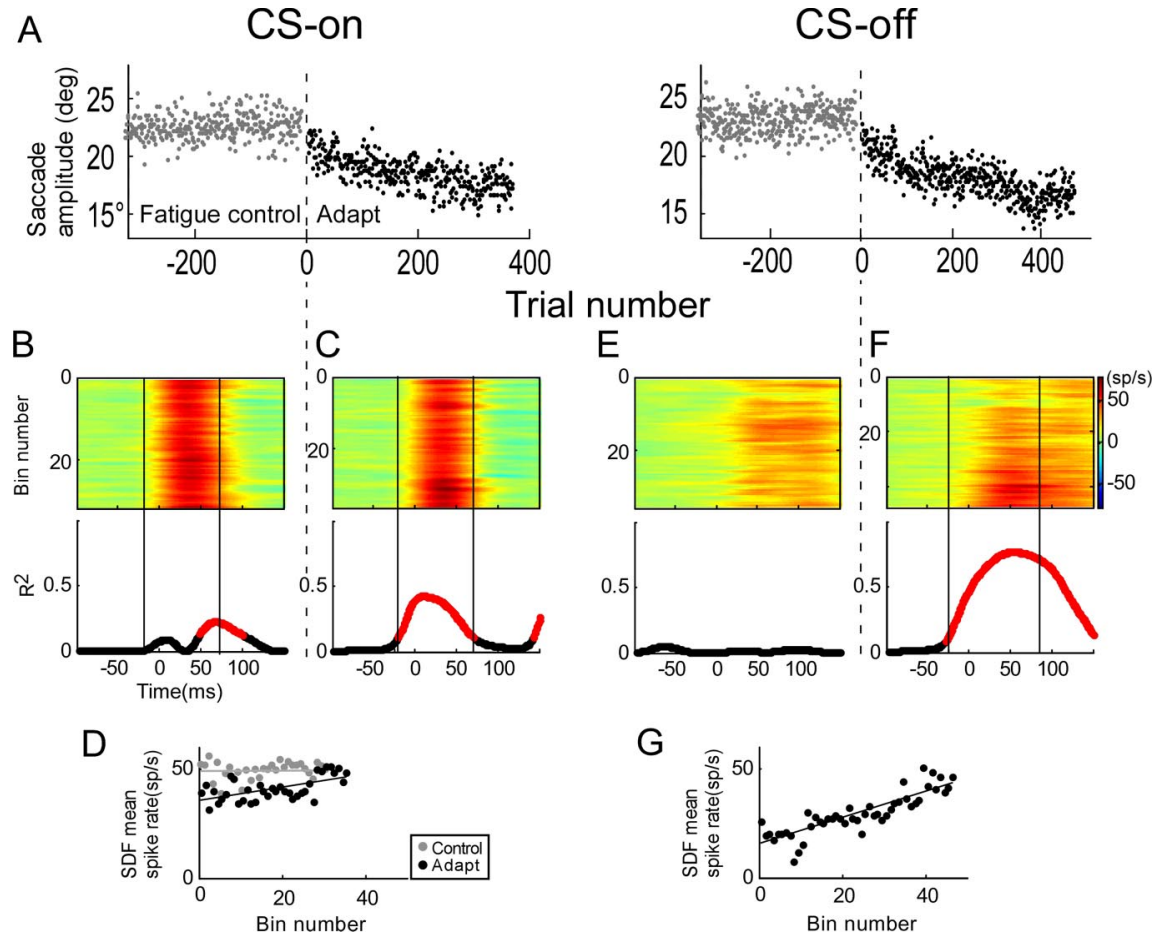

Figure 11. Comparison of the relationship of SS activity with trial number during adaptation and during a fatigue control. CS-on and -off directions were 270 and $90^{\circ}$, respectively. $A$, Saccade amplitude versus trial number during fatigue controls (gray dots from -300 to 0 ) and adaptation (black dots from 0 to $\sim 400$ ). $\boldsymbol{B}-\boldsymbol{F}$, Colorized SS activity presented in order of occurrence and aligned on saccade onset (top panels) and $R^{2}$ of the relationship between $S S$ activity and bin number for every millisecond time slice (bottom panels) for fatigue controls $(\boldsymbol{B}, \boldsymbol{E})$ and adaptations $(\boldsymbol{C}, \boldsymbol{F})$ in the $(S-$ on and -off directions. The vertical black solid lines indicate the analysis intervals. $\boldsymbol{D}, \mathbf{G}$, Average spike rate as a function of bin number for the CS-on and -off directions, respectively. In the CS-on direction, average spike rate increased significantly during adaptation (slope, $0.31 ; p<0.31 ; r=0.59$ ), but not in the fatigue control $(p>0.05)$. In the CS-off direction, average spike rate increased significantly only during adaptation (slope, $0.60 ; p<0.05 ; r=0.86)$. and on its axes and constituted $42 \%$ of the neurons that displayed a functional adaptation signal (Fig. $10 \mathrm{~B}$, fourth quadrant bar).

During adaptation, $\mathrm{P}$-cells with a functional adaptation signal (Fig. 10B, twothirds of all the cells in quadrants 1 through 4) undergo a change in the slope of their firing rate versus amplitude regression. This result suggests that these P-cells could be one candidate site for saccade plasticity and could provide a signal that helps drive saccade adaptation. The remaining one-third of our P-cells did change their activity during adaptation but did not exhibit a functional adaptation signal (i.e., the saccade amplitude sensitivity was the same before and during adaptation). Nevertheless, they also could contribute a cerebellar signal appropriate to help produce the adapted saccade. In this case, we feel that the motor learning probably occurred upstream of the P-cell, and the P-cell simply transmitted changes that had occurred elsewhere.

Previous studies have revealed changes in saccade-related activity during amplitude decrease adaptation in two groups of neurons downstream of oculomotor P-cells. For ipsiversive saccades, cFN neurons (those in the left cFN in Fig. 1) showed either decreases in burst latency $[\sim 60 \%$ of cells estimated from Scudder and McGee (2003)] or increases in the number of spikes (Inaba et al., 2003). Either change would cause contralateral (right side) IBNs to provide either earlier or stronger inhibition, which would reduce the size of leftward saccades. Indeed, both an increase in firing and decrease in burst latency does occur for $48 \%$ of IBNs during contraversive amplitude decrease adaptation [Kojima et al. (2008), their Table 1, 14 of 29]. If these two studies are considered with ours, there is at least a subset of neurons at all stages in the well established cerebellar and brainstem saccade pathways that exhibit changes in discharge appropriate to produce amplitude decrease adaptation.

A more liberal interpretation of the schematic in Figure 1 would suggest that, as long as the net influence of EBN and IBN inputs decreases motoneuron activity, saccade size would decrease. In this case, we would assume that opposite sides of the OMV contribute differential downstream activity with similar weights. Therefore, all P-cells below the line of unity slope in Figure $10 \mathrm{~A}$, which have a greater functional adaptation signal in the CS-on than -off direction, would produce the desired net influence. Consequently, 
to the 16 already identified above, we could add $3 \mathrm{P}$-cells from the third quadrant and 1 from the first quadrant for a total of 20 (53\%) P-cells with potentially appropriate behaviors.

Our estimation that $53 \%$ of P-cells have behavior appropriate to drive adaptation is based exclusively on uncrossed projections of OMV P-cells to the cFN (Fig. 1). However, a brief report has also described some crossed projections (Cherny et al., 2005). If the crossed connections emanate from our second quadrant category of P-cells (Fig. $10 \mathrm{~B}$ ), they also would have the correct sign to participate in the scenario detailed in Figure 1.

The percentage of $\mathrm{P}$-cells in the four categories with responses unique to adaptation differed in experiments using large and small initial target steps (Fig. $10 \mathrm{~B}$ ). In particular, adaptations after small target steps $\left(12,15^{\circ}\right)$ produced changes appropriate for the model in Figure 1 in $58 \%$ of those experiments but in only $14 \%$ of the experiments using large $\left(20,25^{\circ}\right)$ target steps. This difference cannot be explained by monkey-to-monkey variability because the difference in percentages was comparable in monkey $B$ in which we used target steps of both sizes ( 80 vs 15\%). Nor is it likely the result of the small difference in the percentages of amplitude reduction produced after large $(\sim 17.6 \%)$ and small amplitude $(\sim 14.9 \%)$ target steps. However, although the percentage changes were comparable in the two conditions, the postsaccadic errors after large targets were greater. Perhaps these data suggest that different adaptation strategies are used to correct large and small errors.

\section{Comparison with previous studies}

Catz et al. (2008) also have demonstrated that SS activity in the oculomotor vermis changes during adaptation that reduces saccade amplitude. However, they examined only P-cells that discharged a burst of SS activity with saccades, whereas we also considered those that paused and had mixed burst-pause patterns. Indeed, in our sample, pure bursting neurons constituted the distinct minority. In addition, they concentrated on the timing of the population burst, revealing that neither saccade duration nor burst duration changed even though adaptation had reduced saccade amplitude. Moreover, they examined changes in SS activity in the preferred SS burst direction, whereas we considered SS changes in the preferred CS direction, in which we thought that the greatest influence of CSs on SSs was most likely to occur.

Despite these methodological differences, some aspects of the data sets can be compared. Both studies showed that many P-cells showed changes during adaptation that exceeded those that could be accounted for by an underlying relationship with saccade amplitude. Also, we both reported that some P-cells with no or little phasic activity before adaptation can acquire some during adaptation. However, Catz et al. (2008) found only decreases in burst rate during adaptation, whereas some of our P-cells showed increasing burst activity. In our study, some of the differences in firing patterns could be rationalized by considering the recording side, which was not reported in their study. Finally, we showed that P-cells with a pause in SS activity also often showed changes that would be appropriate to produce the changes in cFN activity that have been reported during adaptation. Indeed, $62 \%$ of all our cells showed changes specific to adaptation, whereas $64 \%$ of their bursting P-cells did.

Modification of SS activity also occurs during adaptation of other voluntary targeting movements. These include smoothpursuit eye movements adapting to double velocity steps (Kahlon and Lisberger, 2000; Medina and Lisberger, 2008, 2009) and horizontal arm movements adapting to a manipulandum with al- tered movement "gains" (Ojakangas and Ebner, 1992) or subjected to changing loads (Gilbert and Thach, 1977). For both smooth-pursuit eye movements and arm movements, approximately one-third of the involved P-cells exhibited adaptationrelated changes in SS activity. Therefore, our finding that only a subset of vermis P-cells appears to drive saccade motor adaptation is not unusual. At least $42 \%$ of the P-cell firing patterns were appropriate to participate specifically in adaptation based on our current knowledge of oculomotor cerebellar anatomy. Future studies may refine this model so that P-cells with apparently inappropriate changes in SS activity also would be implicated in saccade adaptation.

\section{References}

Albus JS (1971) A theory of cerebellar function. Math Biosci 10:25-61.

Barash S, Melikyan A, Sivakov A, Zhang M, Glickstein M, Thier P (1999) Saccadic dysmetria and adaptation after lesions of the cerebellar cortex. J Neurosci 19:10931-10939.

Catz N, Dicke PW, Thier P (2008) Cerebellar-dependent motor learning is based on pruning a Purkinje cell population response. Proc Natl Acad Sci U S A 105:7309-7314.

Cherny E, Galloway E, Kaneko CRS, Noto CT, Robinson FR (2005) Bilateral projection from the oculomotor vermis to the caudal fastigial nucleus in monkey. Soc Neurosci Abstr 31:755.3.

Fuchs AF, Robinson DA (1966) A method for measuring horizontal and vertical eye movement chronically in the monkey. J Appl Physiol 21:1068-1070.

Fuchs AF, Robinson FR, Straube A (1993) Role of the caudal fastigial nucleus in saccade generation. I. Neuronal discharge patterns. J Neurophysiol 70:1723-1740.

Gilbert PF, Thach WT (1977) Purkinje cell activity during motor learning. Brain Res 128:309-328.

Golla H, Tziridis K, Haarmeier T, Catz N, Barash S, Thier P (2008) Reduced saccadic resilience and impaired saccadic adaptation due to cerebellar disease. Eur J Neurosci 27:132-144.

Hopp JJ, Fuchs AF (2004) The characteristics and neuronal substrate of saccadic eye movement plasticity. Prog Neurobiol 72:27-53.

Inaba N, Iwamoto Y, Yoshida K (2003) Changes in cerebellar fastigial burst activity related to saccadic gain adaptation in the monkey. Neurosci Res 46:359-368.

Judge SJ, Richmond BJ, Chu FC (1980) Implantation of magnetic search coils for measurement of eye position: an improved method. Vision Res 20:535-538.

Kahlon M, Lisberger SG (2000) Changes in the responses of Purkinje cells in the floccular complex of monkeys after motor learning in smooth pursuit eye movements. J Neurophysiol 84:2945-2960.

Kojima Y, Iwamoto Y, Robinson FR, Noto CT, Yoshida K (2008) Premotor inhibitory neurons carry signals related to saccade adaptation in the monkey. J Neurophysiol 99:220-230.

Marr D (1969) A theory of cerebellar cortex. J Physiol (Lond) 202:437-470.

Martin TA, Keating JG, Goodkin HP, Bastian AJ, Thach WT (1996) Throwing while looking through prisms. I. Focal olivocerebellar lesions impair adaptation. Brain 119:1183-1198.

McLaughlin S (1967) Parametric adjustment in saccadic eye movements. Percept Psychophys 2:359-362.

Medina JF, Lisberger SG (2008) Links from complex spikes to local plasticity and motor learning in the cerebellum of awake-behaving monkeys. Nat Neurosci 11:1185-1192.

Medina JF, Lisberger SG (2009) Encoding and decoding of learned smoothpursuit eye movements in the floccular complex of the monkey cerebellum. J Neurophysiol 102:2039-2054.

Moschovakis AK, Scudder CA, Highstein SM (1996) The microscopic anatomy and physiology of the mammalian saccadic system. Prog Neurobiol 50:133-254.

Noda H, Sugita S, Ikeda Y (1990) Afferent and efferent connections of the oculomotor region of the fastigial nucleus in the macaque monkey. J Comp Neurol 302:330-348.

Ohtsuka K, Noda H (1991) Saccadic burst neurons in the oculomotor region of the fastigial nucleus of macaque monkeys. J Neurophysiol 65:1422-1434.

Ohtsuka K, Noda H (1995) Discharge properties of Purkinje cells in the 
oculomotor vermis during visually guided saccades in the macaque monkey. J Neurophysiol 74:1828-1840.

Ojakangas CL, Ebner TJ (1992) Purkinje cell complex and simple spike changes during a voluntary arm movement learning task in the monkey. J Neurophysiol 68:2222-2236.

Optican LM, Robinson DA (1980) Cerebellar-dependent adaptive control of primate saccadic system. J Neurophysiol 44:1058-1076.

Robinson DA (1963) A method of measuring eye movement using a scleral search coil in a magnetic field. IEEE Trans Biomed Electron 10:137-145.

Scudder CA, McGee DM, Balaban CD (2000) Connections of monkey saccade-related fastigial nucleus neurons revealed by anatomical and physiological methods. Soc Neurosci Abstr 26:363.18.

Scudder CA, McGee DM (2003) Adaptive modification of saccade size produces correlated changes in the discharges of fastigial nucleus neurons. J Neurophysiol 90:1011-1026.
Scudder CA, Kaneko CS, Fuchs AF (2002) The brainstem burst generator for saccadic eye movements. A modern synthesis. Exp Brain Res 142:439-462.

Soetedjo R, Fuchs AF (2006) Complex spike activity of Purkinje cells in the oculomotor vermis during behavioral adaptation of monkey saccades. J Neurosci 26:7741-7755.

Soetedjo R, Kaneko CR, Fuchs AF (2002) Evidence against a moving hill in the superior colliculus during saccadic eye movements in the monkey. J Neurophysiol 87:2778-2789.

Soetedjo R, Kojima Y, Fuchs AF (2008) Complex spike activity in the oculomotor vermis of the cerebellum: a vectorial error signal for saccade motor learning? J Neurophysiol 100:1949-1966.

Yamada J, Noda H (1987) Afferent and efferent connections of the oculomotor cerebellar vermis in the macaque monkey. J Comp Neurol 265: $224-241$. 